\title{
Heurísticas para Visualização de Dados
}

\author{
Fernanda C. Ribeiro ${ }^{1}$, Bárbara P. Caetano ${ }^{1}$, Melise M. V. de Paula ${ }^{1}$, Miriam B. F. \\ Chaves $^{2}$, Vinicius de F. Silva ${ }^{2}$, Sérgio A. Rodrigues ${ }^{3}$, Jano M. de Souza ${ }^{3}$ \\ ${ }^{1}$ Instituto de Ciências Exatas - Universidade Federal de Itajubá (UNIFEI) \\ Av. BPS, 1303 - Bairro Pinheirinho - 37500-903 - Itajubá - MG - Brasil. \\ ${ }^{2}$ Ministério do Planejamento, Orçamento e Gestão \\ Esplanada dos Ministérios - Brasília - DF - Brasil \\ ${ }^{3}$ Programa de Engenharia de Sistemas e Computação - COPPE/UFRJ \\ Caixa Postal: 68511 - 21941-972 - Rio de Janeiro - RJ - Brasil. \\ \{fernandafcrk, bpimentacaetano, melisepaula, miriambfc\} @gmail.com, \\ \{vinicius.faria-silva\}@planejamento.gov.br, \{sergio, jano\}@cos.ufrj.br
}

\begin{abstract}
Information Systems are used to collect, store and disseminate information generated by users. Moreover, these systems help in decision making process as well as the Information Visualization (InfoVis) techniques. Experts recommend the use of heuristics to facilitate the selection process of InfoVis techniques to be applied in any context. The goal of this work was analyze the literature on InfoVis and transparency of public data in order to identify a heuristic set which could be used to verify the suitability of application of visualization techniques in the government context.
\end{abstract}

Resumo. Os Sistemas de Informação são utilizados para coletar, armazenar e disseminar as informações geradas pelos usuários. Além disso, esses sistemas auxiliam no processo de tomada de decisão assim como as técnicas de Visualização de Informação (InfoVis). Especialistas recomendam o uso de heurísticas para facilitar o processo de escolha das técnicas de InfoVis a serem aplicadas em qualquer contexto. O objetivo deste trabalho foi analisar a literatura sobre InfoVis e a transparência dos dados públicos de forma a identificar um conjunto de heurísticas que pudessem ser utilizadas para verificar a adequação da aplicação das técnicas de visualização no contexto governamental.

\section{Introdução}

O avanço tecnológico está modificando a maneira como os usuários se comunicam, interagem, obtêm dados e informações e tomam decisões. Neste cenário, os Sistemas de Informação (SI) são utilizados para coletar, manipular, armazenar, transmitir, recuperar e disseminar os dados e as informações geradas pelos usuários (Souza, 2009). Além de apresentar essas funcionalidades, todo sistema de informação deve ser útil e apresentar uma boa interatividade com as pessoas (Sorj, 2003).

A Visualização de Informação (InfoVis) é uma área de estudo que tem sido muito utilizada para aperfeiçoar algumas funcionalidades dos sistemas de informação em diferentes áreas. Para Luzzardi (2010), um sistema de InfoVis é um conjunto de uma 
ou mais técnicas de visualização que geram representações visuais de um ou mais conjuntos de dados. O objetivo destes sistemas é permitir que o usuário analise, interprete e manipule grandes conjuntos de dados com o auxílio de mecanismos de interação (Luzzardi, 2010). Os sistemas de InfoVis podem ser aplicados em diversos contextos. No contexto governamental, por exemplo, a InfoVis pode ser utilizada para representar os dados públicos, auxiliar a sociedade na compreensão dos dados disponibilizados e alavancar a transparência das informações (Paula et. al 2011). No setor governamental, a transparência é definida por Silva (2010) como estratégias que permitam que o cidadão tome consciência das ações governamentais. Além disso, a transparência está relacionada ao entendimento das formas de disponibilização dos dados governamentais por parte dos órgãos públicos (Silva, 2010).

Contudo, ainda que os dados estejam disponíveis, o usuário deve encontrá-los com facilidade. Ou seja, o sistema utilizado para publicar os dados deve possuir mecanismos que auxiliem no acesso às informações atendendo às necessidades dos usuários. Para verificar se os SI são úteis, apresentam boa interatividade e se atendem às necessidades dos usuários, especialistas recomendam o uso de avaliações. Segundo Valiati (2008), um dos métodos de avaliação mais conhecidos e utilizados na inspeção de interfaces é o método heurístico. A avaliação heurística consiste em usuários experientes avaliarem e julgarem se o sistema atende às heurísticas predefinidas (Forsell e Johansson, 2010). Avaliações heurísticas também são aplicadas em sistemas de InfoVis.

O objetivo deste trabalho foi analisar a literatura sobre InfoVis, transparência dos dados públicos e heurísticas para InfoVis de forma a identificar um conjunto de heurísticas que pudessem ser utilizadas para verificar a adequação da aplicação das técnicas de visualização no contexto governamental. Vale ressaltar que, este trabalho está associado a um projeto de pesquisa (VisPublica) que tem por objetivo analisar as técnicas de visualização para o contexto governamental. As heurísticas definidas neste trabalho não se aplicam exclusivamente aos dados governamentais abertos. Contudo, o cenário governamental foi considerado de forma a evidenciar até que ponto as técnicas de InfoVis influenciam a percepção dos usuários. Neste caso, a transparência dos dados apresentados foi um dos aspectos analisados.

O texto está dividido em 5 seções. Após a introdução é apresentada a revisão da literatura considerando alguns trabalhos relacionados às heurísticas para InfoVis. Na terceira seção, é apresentado o conjunto de heurísticas identificado pelos autores. Os resultados do experimento realizado para validar as heurísticas são apresentados na seção 4. Por fim, na quinta seção, estão as considerações finais.

\section{Revisão da Literatura}

Segundo Nielsen e Mack (1994), a avaliação heurística é um método de avaliação da área de IHC (Interação Humano Computador). Através desse método, usuários experientes na área de usabilidade avaliam sistemas e julgam se os mesmos atendem aos objetivos das diretrizes (heurísticas) predefinidas (Forsell e Johansson, 2010). O método de avaliação heurística é reconhecido pela comunidade de InfoVis (Zuk et. al, 2006). Tory e Moller (2005) recomendam o uso da avalição heurística para analisar as técnicas de visualização. Segundo Zuk et. al (2006), este processo é fortemente influenciado pela 
escolha das heurísticas e dos avaliadores. Para Forsell e Johansson (2010), o conjunto de heurísticas para InfoVis deve ser geral o suficiente para ser usado para qualquer técnica de visualização e ser fácil de entender e aplicar. Forsell (2012) caracterizou o método de avaliação heurística como rápido, com baixo custo e relativamente fácil de utilizar, possibilitando gerar resultados considerados significantes.

Forsell e Johansson (2010) definiram um conjunto de heurísticas para avaliar técnicas de InfoVis. Para definir este conjunto, os autores realizaram experimentos com o auxílio de seis especialistas na área de InfoVis e/ou IHC. No estudo, os participantes avaliaram os 74 problemas de usabilidade utilizando seis conjuntos de heurísticas. Esses problemas estavam relacionados, por exemplo, à representação visual, aos mecanismos de interação e à facilidade e suporte na análise e exploração dos dados. Cada heurística foi classificada em uma escala de 0 a 5, onde (Forsell e Johansson, 2010):

- 0 = a heurística não auxilia na solução do problema de usabilidade.

- 1 = a heurística aborda superficialmente alguns aspectos que explicam o problema de usabilidade.

- 2 = a heurística resolve uma pequena parte do problema, mas há aspectos importantes do problema que não são esclarecidos pela heurística.

- $\quad 3$ = a heurística resolve grande parte do problema de usabilidade, mas ainda há aspectos que não são explicados.

- 4 = a heurística esclarece quase que completamente o problema de usabilidade, no entanto há aspectos do problema que não são solucionados pela heurística.

- 5 = a heurística auxilia na solução de todo o problema de usabilidade.

As heurísticas que receberam nota entre 3 e 5 foram selecionadas para compor o novo conjunto de heurísticas definido por Forsell e Johansson (2010). A seguir serão apresentadas as heurísticas deste trabalho.

\section{Conjunto de heurísticas para visualização de dados}

Nesta seção, antes de apresentar as heurísticas, serão detalhados os critérios de avaliação utilizados para defini-las. Esses critérios estão subdivididos em 3 classes. Na primeira classe, são consideradas as características estáticas da representação visual. A segunda classe é voltada para a avaliação dos mecanismos de interação. Por fim, como uma das perspectivas consideradas neste trabalho é a transparência de dados públicos, na terceira classe, são apresentados os critérios de avaliação relacionados à transparência de dados.

\subsection{Critérios de avaliação para a representação visual}

O primeiro critério relacionado à representação visual é o Volume de Dados. Algumas técnicas de visualização procuram representar todo o conjunto de dados em uma única representação visual. No entanto, em alguns casos, não é possível representar toda informação de forma adequada por causa dos problemas relacionados ou à oclusão dos dados, ou ao espaço insuficiente, ou a desordem visual (Luzzardi, 2003).

Segundo Valiati (2008), quando ocorrem, por exemplo, os problemas de oclusão e espaço insuficiente, a técnica de visualização deve disponibilizar recursos interativos 
que possibilitem e auxiliem a análise dos dados (exemplo de mecanismos de interação: zoom, rotação ou distorção).

O segundo critério utilizado para avaliar a representação visual é o Uso de Cores. Para Tufte (2001), o uso de cores em visualizações tem por objetivo rotular, medir, representar ou imitar a realidade, animar ou decorar. Segundo Few (2008), cores diferentes devem ser usadas apenas quando essas cores evidenciarem significados à visualização auxiliando o usuário na interpretação dos dados.

O Uso de Rótulos também auxilia o usuário no processo de análise dos dados (Valiati, 2008). Este terceiro critério não está associado apenas ao uso de legendas. Segundo Yau (2010), se o gráfico utilizar os eixos coordenados X e Y, então esses eixos também devem ser rotulados. Além dos eixos, os elementos da visualização também podem estar rotulados.

\subsection{Critérios de avaliação para os mecanismos de interação}

Segundo Valiati (2008), os mecanismos de interação contribuem para a compreensão dos dados representados. Para Luzzardi (2003), as técnicas de visualização devem fornecer mecanismos rápidos e eficazes que permitem ao usuário encontrar (buscar) informações através de consultas dinâmicas e eficientes. Já para Yamaguchi (2010), através de recursos interativos como, por exemplo, a filtragem, o usuário pode eliminar os elementos da visualização que não interessam no momento da análise.

Valiati (2008) afirma que a técnica de visualização também deve permitir que o usuário selecione os elementos da representação visual. Além disso, a seleção deve ser mantida enquanto o usuário executa as operações necessárias durante o processo de análise e os elementos selecionados devem ser destacados dos demais para que o usuário identifique a seleção (Valiati, 2008; Luzzardi, 2003).

\subsection{Critérios de avaliação para transparência dos dados}

Em Aló (2009), a autora define um Catálogo de Transparência que é um conjunto de operacionalizações que guia uma organização na implementação da transparência através do fornecimento de informações sobre suas atividades e ações realizadas. No catálogo, foram identificados critérios que contribuem para a avaliação da transparência dos dados. Neste trabalho, estes critérios foram adaptados de forma a contemplar aspectos relacionados à InfoVis. Os critérios considerados são: adaptabilidade, clareza, amigabilidade e intuitividade.

Aló (2009) define adaptabilidade como a capacidade de mudar de acordo com as circunstâncias e necessidades. No contexto da InfoVis, entende-se que a adaptabilidade é a capacidade de mudar a visualização de acordo com as necessidades do usuário durante a análise dos dados. Esse critério é aplicado às técnicas com recurso interativo. Para Aló (2009), clareza está relacionada à capacidade de nitidez e compreensão. O objetivo principal da InfoVis e consequentemente das técnicas de visualização é facilitar o processo de compreensão dos dados. Logo, o critério de clareza, no contexto da InfoVis, está relacionado à capacidade da visualização tornar os dados compreensíveis ao usuário. 
Amigabilidade é a capacidade de utilização da visualização sem esforço (Aló, 2009). No contexto deste trabalho, uma visualização é considerada amigável quando o usuário utiliza os recursos interativos da técnica com o mínimo de esforço durante a execução das tarefas. Vale ressaltar que para técnicas que não possuem recursos interativos, os critérios Clareza e Amigabilidade se sobrepõem. Mas para as técnicas com recursos interativos, esses critérios são diferentes.

Intuitividade é a capacidade de ser utilizado sem aprendizado prévio (Aló, 2009). No contexto da InfoVis, caso a representação visual seja interativa, este critério avalia se a interação do usuário com a visualização é intuitiva ou se exige o mínimo de aprendizado para o usuário utilizar estes recursos.

\subsection{Conjunto de heurísticas}

A partir dos critérios apresentados nas subseções anteriores foram definidas heurísticas para analisar a adequação das técnicas de visualização disponíveis ao contexto governamental. A cada heurística, estão associados os critérios de avaliação das subseções 3.1, 3.2 e 3.3, conforme mostra a tabela 1 .

Tabela 1. Heurísticas para Visualização de Dados

\begin{tabular}{|l|l|}
\hline \multicolumn{1}{|c|}{ Heurística } & Critérios de Avaliação \\
\hline $\begin{array}{l}\text { H1. Caso a representação visual apresente algum problema } \\
\text { relacionado ao volume de dados, a técnica deve permitir a } \\
\text { utilização de recursos interativos para minimizar o problema } \\
\text { apresentado. }\end{array}$ & $\begin{array}{l}\text { Volume de Dados; } \\
\text { Adaptabilidade; } \\
\text { Amigabilidade }\end{array}$ \\
\hline $\begin{array}{l}\text { H2. Os recursos interativos devem ser facilmente } \\
\text { identificados. }\end{array}$ & Intuitividade \\
\hline $\begin{array}{l}\text { H3. A técnica visualização deve fornecer ajuda quanto ao uso } \\
\text { dos mecanismos de interação. }\end{array}$ & $\begin{array}{l}\text { Amigabilidade; } \\
\text { Intuitividade }\end{array}$ \\
\hline $\begin{array}{l}\text { H4. A técnica de visualização deve permitir a seleção de } \\
\text { elementos na visualização. }\end{array}$ & $\begin{array}{l}\text { Seleção; Filtragem } \\
\text { Adaptabilidade }\end{array}$ \\
\hline $\begin{array}{l}\text { H5. A técnica de visualização deve permitir a realização de } \\
\text { consultas, ou seja, a localizção de elementos na visualização. }\end{array}$ & Busca; Adaptabilidade \\
\hline $\begin{array}{l}\text { H6. A técnica de visualização deve permitir a utilização } \\
\text { adequada de cores para destacar os elementos da visualização. }\end{array}$ & $\begin{array}{l}\text { Uso de Cores; Clareza; } \\
\text { Adaptabilidade }\end{array}$ \\
\hline $\begin{array}{l}\text { H7. A técnica de visualização deve apresentar rótulos nos } \\
\text { elementos da representação visual para que o usuário } \\
\text { compreenda a informação representada. }\end{array}$ & $\begin{array}{l}\text { Uso de Rótulos; } \\
\text { Clareza; } \\
\text { Adaptabilidade }\end{array}$ \\
\hline
\end{tabular}

Definido o conjunto de heurística, o passo seguinte deste trabalho foi a realização de experimentos para validar as heurísticas. O procedimento para a realização do experimento bem como os resultados obtidos serão explicados na seção seguinte.

\section{Experimento}

O objetivo do experimento foi validar o conjunto de heurísticas apresentado na seção anterior. A proposta foi apresentar a um grupo de participantes visualizações que atendiam e outras que não atendiam às heurísticas descritas e obter a opinião do usuário quanto à utilidade da visualização. Esperava-se que, ao fim do experimento, as 
visualizações que atendessem às heurísticas fossem consideradas pelo usuário mais úteis no contexto apresentado.

O experimento foi realizado em 2 etapas: coleta de dados sobre o perfil do usuário e análise das visualizações. Na primeira etapa, os participantes foram orientados em relação ao objetivo do experimento e à sequência das atividades que deveriam ser executadas tendo sido coletados alguns dados a respeito do perfil. $\mathrm{Na}$ segunda etapa, foram apresentadas 4 visualizações aos participantes, uma de cada vez. A cada visualização, eram apresentadas informações sobre um determinado conjunto de dados e duas tarefas. Estas tarefas estavam relacionadas à verificação de valores associados a um determinado estado e período de tempo. Além disso, o participante deveria analisar as sentenças relacionadas às heurísticas e escolher um dos seguintes valores: concordo, concordo com restrições ou discordo. Também foi disponibilizado ao participante um espaço para que o mesmo colocasse sugestões ou comentários (restrições) em relação às sentenças.

Para a realização do experimento foi desenvolvida uma aplicação web. As páginas da aplicação foram definidas em função das etapas do experimento. Os participantes acessaram a aplicação através de um link enviado por email. As visualizações do experimento foram implementadas utilizando bibliotecas de visualização.

Para a Visualização 1 foi utilizada a técnica Gráfico com Animação, conforme mostra a Figura 1. O conjunto de dados escolhido para ser representado pela Visualização 1 se refere aos recursos do FGTS (Fundo de Garantia de Tempo de Serviço) aplicados em habitação entre os anos de 1995 e 2009 (Serie 1, 2012). As tarefas solicitadas para o usuário executar foram: verificar o valor do FGTS no estado de Minas Gerais nos anos de 1995 e 2006 e verificar qual estado possuía o maior investimento do FGTS no ano de 2002.

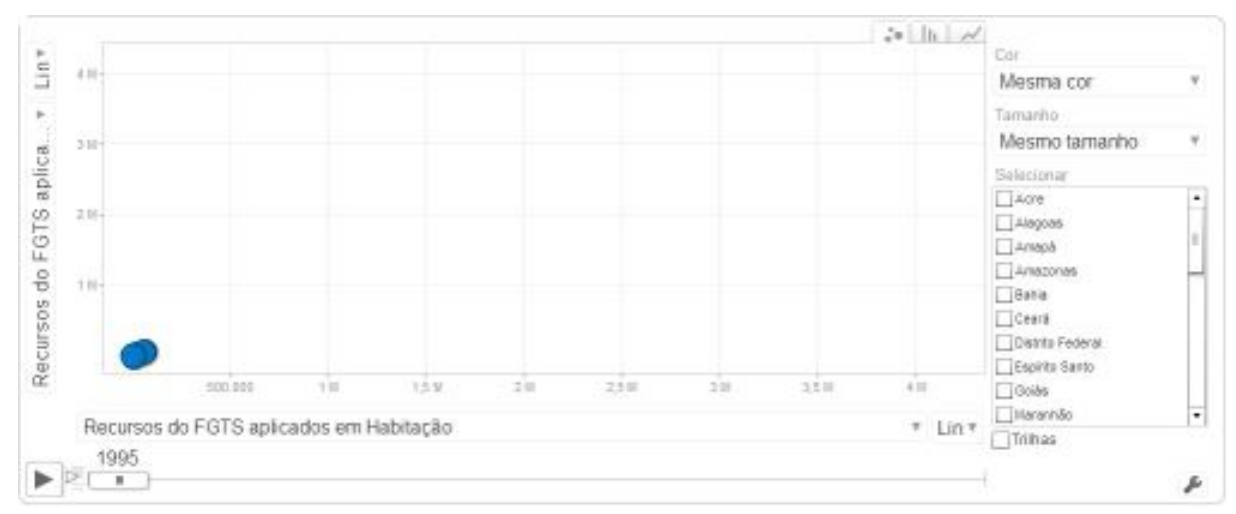

Figura 1. Gráfico com Animação.

A Visualização 1 apresentava problemas relacionados à oclusão dos dados relacionados à sobreposição de círculos. Contudo, recursos interativos poderiam ser utilizados para solucionar este problema. Embora a representação visual disponibilizasse mecanismos de interação, alguns recursos (como por exemplo, o recurso de zoom) não eram intuitivos.

A Visualização 2 foi gerada com a técnica Gráfico de Colunas para representar o conjunto de dados referente à quantidade de escolas beneficiadas com o programa escola 
aberta (Serie 2, 2012). Esta visualização possuía mecanismos de interação e instruções sobre como utilizar estes recursos. Além disto, a Visualização 2 atendia a maioria das heurísticas descritas: H1, H2, H3, H6 e H7.

A técnica Gráfico de Bolhas foi utilizado nas visualizações 3 e 4 . O conjunto de dados escolhido se refere às pessoas atendidas pelo programa Luz Para Todos (Serie 3, 2012). A Visualização 3 atendia a todas as heurísticas. A Visualização 4 apresentava problemas relacionados ao grande volume de dados, mas não disponibilizava recursos interativos que pudessem auxiliar na solução dos problemas.

As características das técnicas descritas acima não foram apresentadas aos participantes para não influenciar os resultados do experimento. Estes resultados são apresentados na subseção seguinte.

\subsection{Resultados obtidos}

Vinte e uma pessoas participaram do experimento, sendo 11 mulheres e 10 homens, com idade entre 21 e 50 anos. Cerca de $62 \%$ dos participantes estavam cursando algum curso de graduação e $90 \%$ atuavam de alguma forma na área de computação. Quanto ao grau de conhecimento dos participantes sobre InfoVis o resultado foi: $48 \%$ das pessoas afirmaram não ter conhecimento sobre a área de InfoVis, 38\% dos participantes já leram algum trabalho relacionado à InfoVis e $14 \%$ das pessoas trabalhavam na área de InfoVis. A seguir, os resultados serão apresentados de acordo com a heurística relacionada. Para cada heurística, serão referenciadas as sentenças e questionamentos de cada visualização diretamente relacionada à heurística em questão.

Conforme apresentado anteriormente, a Visualização 1 apresentava problemas relacionados à oclusão dos dados. Cerca de $90 \%$ dos participantes concordaram que a sobreposição dos círculos prejudicou a interpretação dos dados. Para solucionar o problema de oclusão, segundo a heurística H1, a técnica de InfoVis deve disponibilizar mecanismos de interação que auxiliem a análise dos dados. Em torno de $71 \%$ dos participantes afirmaram que o recurso de zoom facilitou a interpretação dos dados (conforme mostra a Figura 2). Nenhum participante declarou que o recurso de reprodução dinâmica não facilitou a compreensão dos dados. Cerca de $90 \%$ dos participantes afirmaram que foi fácil interpretar os dados utilizando o recurso que permite a alteração das cores. Estes resultados demonstram que a heurística (H1) é válida.

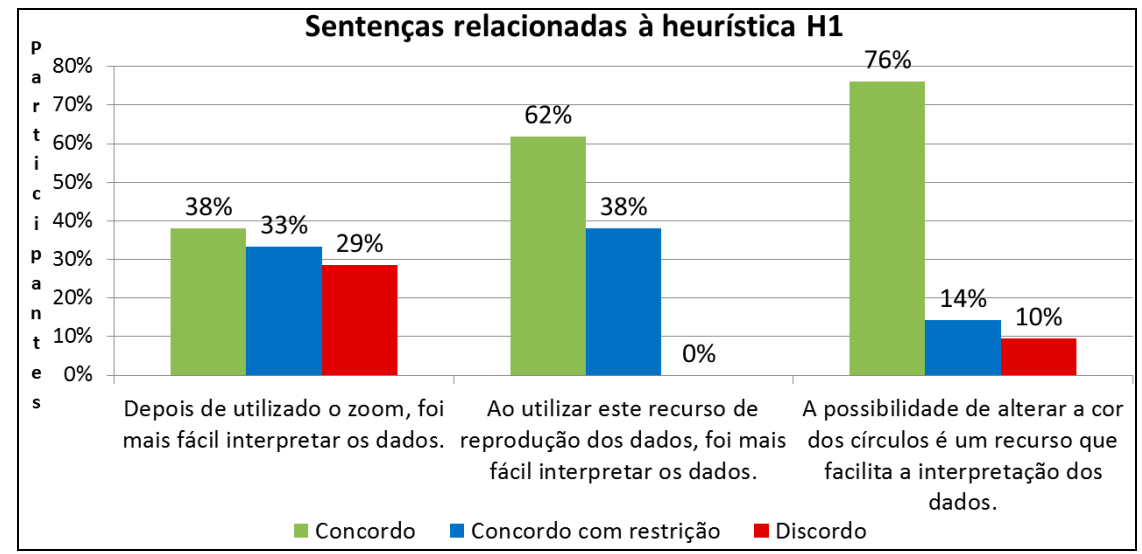

Figura 2. Sentenças relacionadas à heurística $\mathrm{H1}$. 
Segundo a heurística $\mathrm{H} 2$, os mecanismos de interação devem ser facilmente identificados. Os recursos interativos da Visualização 2 foram identificados com facilidade pela maioria dos participantes. Conforme mostra a Figura 3, 76\% identificaram o recurso de zoom com facilidade. Nenhum participante encontrou dificuldades para identificar o recurso que permite a eliminação de elementos da visualização. Além disso, todos participantes concordaram que a facilidade ao identificar esses mecanismos de interação contribuiu com a interpretação dos dados. Logo, esses resultados também contribuem com a afirmação de que a heurística H2 é válida.

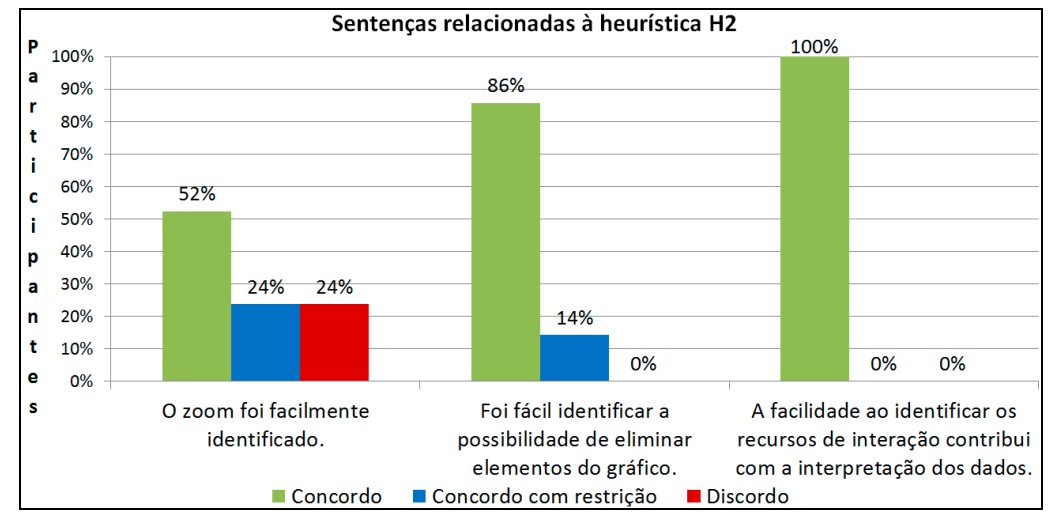

Figura 3. Sentenças relacionadas à heurística H2.

A heurística $\mathrm{H} 3$ afirma que as técnicas de InfoVis devem disponibilizar instruções sobre o uso dos mecanismos de interação. Um participante comentou que se sentiu perdido por não haver instruções de uso na Visualização 1. Além disso, para $81 \%$ das pessoas que analisaram a Visualização 1, seria mais fácil analisar os dados se fossem apresentadas instruções sobre como interagir com a visualização. Já a Visualização 2 apresentava instruções sobre como usar os recursos interativos. Conforme mostra a Figura 4, nenhum participante discordou que a ajuda fornecida na Visualização 2 auxiliou a análise dos dados. Com esses resultados, é possível afirmar que a heurística H3 é válida, pois as instruções de uso permitiram que os participantes analisassem os dados com facilidade.

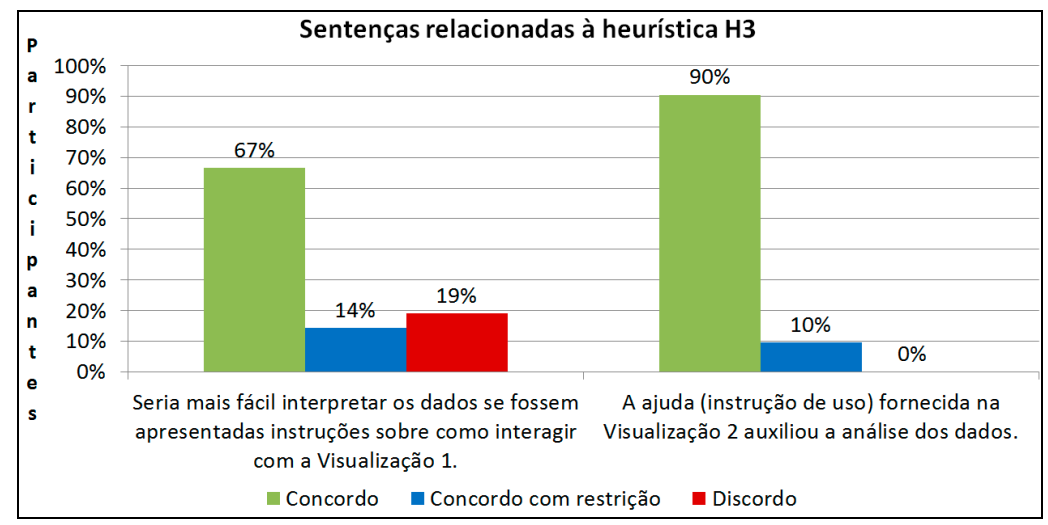

Figura 4. Sentenças relacionadas à heurística H3.

Segundo a heurística $\mathrm{H} 4$, a técnica de InfoVis deve permitir a seleção de elementos da visualização. A Visualização 2 permite que o usuário selecione elementos 
para eliminá-los da representação visual. Conforme mostra Figura 5, nenhum participante encontrou dificuldades para identificar o recurso de eliminação dos elementos. Além disso, todos os participantes afirmaram que este recurso facilitou o processo de análise dos dados. Logo, é possível afirmar que a heurística H4 é coerente.

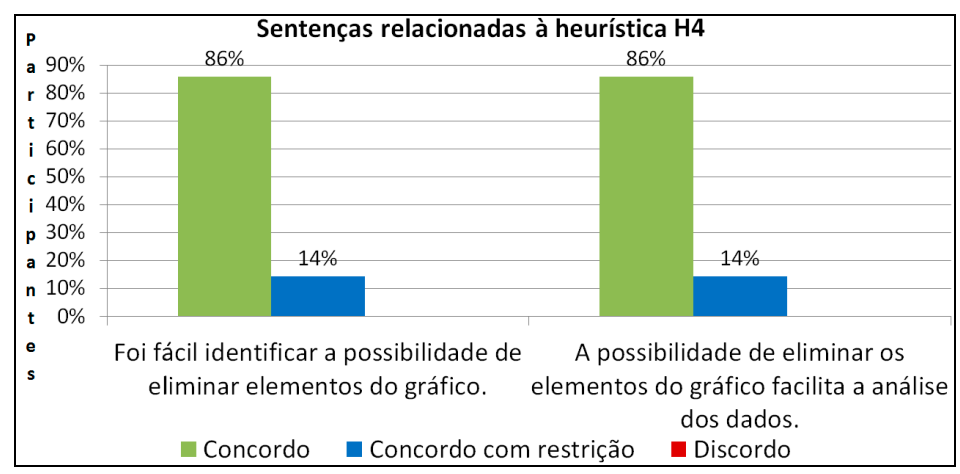

Figura 5. Sentenças relacionadas à heurística $\mathbf{H 4}$.

A heurística H5 recomenda que a técnica de InfoVis permita a localização de elementos na representação visual. De acordo com a Figura 6, para 86\% dos participantes, se a Visualização 1 disponibilizasse o recurso de busca, esse recurso auxiliaria a análise dos dados. Em torno de $76 \%$ e $85 \%$ das pessoas que analisaram a Visualização 2 e 4, respectivamente, concordaram que se o recurso de busca estivesse disponível nessas visualizações, esse recurso facilitaria a interpretação dos dados. Estes resultados sugerem que a heurística H5 é válida, pois os participantes (ao analisarem as sentenças relacionadas a essa heurística) concordaram que a heurística $\mathrm{H} 5$ fez diferença no processo de análise dos dados.

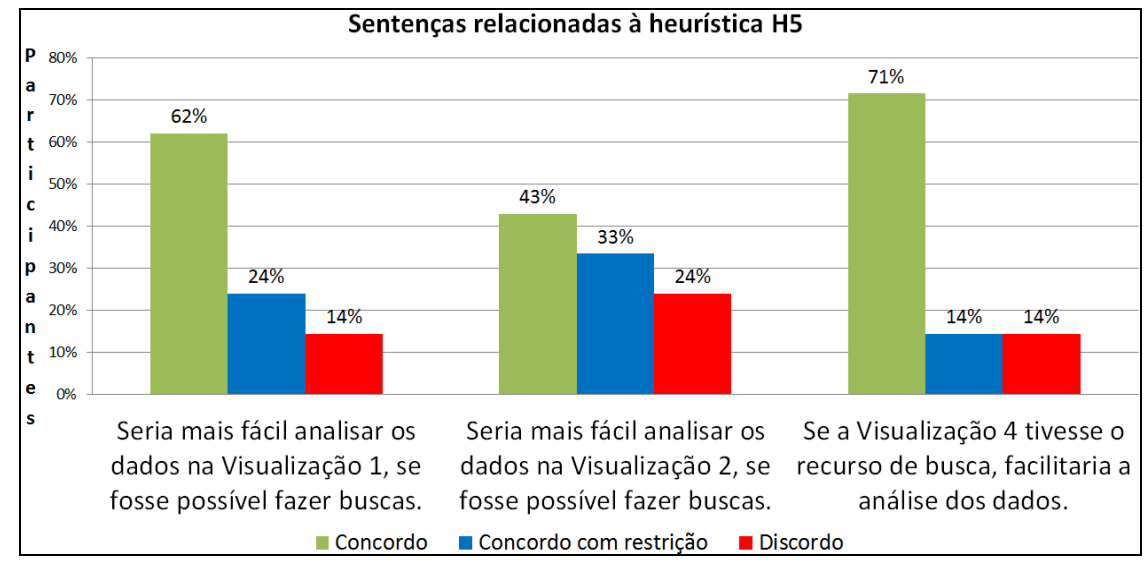

Figura 6. Sentenças relacionadas à heurística H5.

Segundo a heurística H6, a técnica de InfoVis deve permitir a utilização adequada de cores para destacar os elementos da visualização. Para $76 \%$ dos participantes foi fácil interpretar os dados utilizando o recurso da Visualização 1 que permite a alteração de cores. Para $86 \%$ das pessoas que analisaram a Visualização 2 , o uso de diversas cores auxiliou na análise dos dados. Conforme mostra a Figura 7, 81\% dos participantes concordaram que a utilização de cores diferenciadas na Visualização 4 facilitaria a compreensão dos dados. Logo, os resultados obtidos nas 3 visualizações citadas acima permitem afirmar que a heurística H6 é válida, pois a utilização adequada das cores facilitou a interpretação dos dados. 


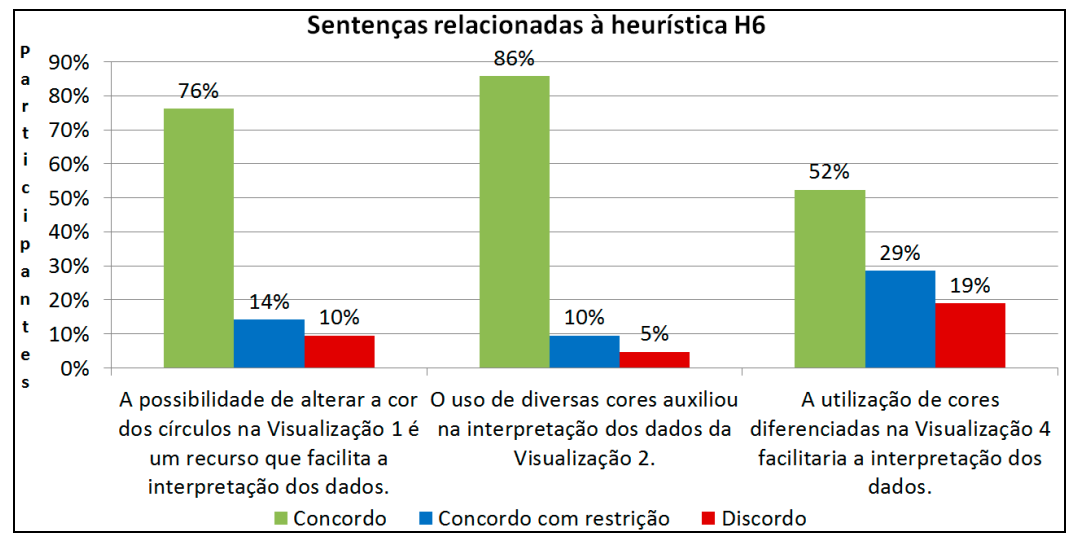

Figura 7. Sentenças relacionadas à heurística H6.

Segundo a heurística H7, a técnica de InfoVis deve apresentar rótulos nos elementos da representação visual para que o usuário compreenda a informação representada. Nenhum participante discordou que os rótulos auxiliaram na compreensão dos dados das visualizações 1 e 2, conforme mostra a Figura 8. Para 71\% dos participantes, os rótulos da visualização 3 auxiliaram na compreensão dos dados. Pelo fato dos participantes concordarem que os rótulos fizeram diferença na análise dos dados, é possível afirmar que a heurística $\mathrm{H} 7$ é coerente.

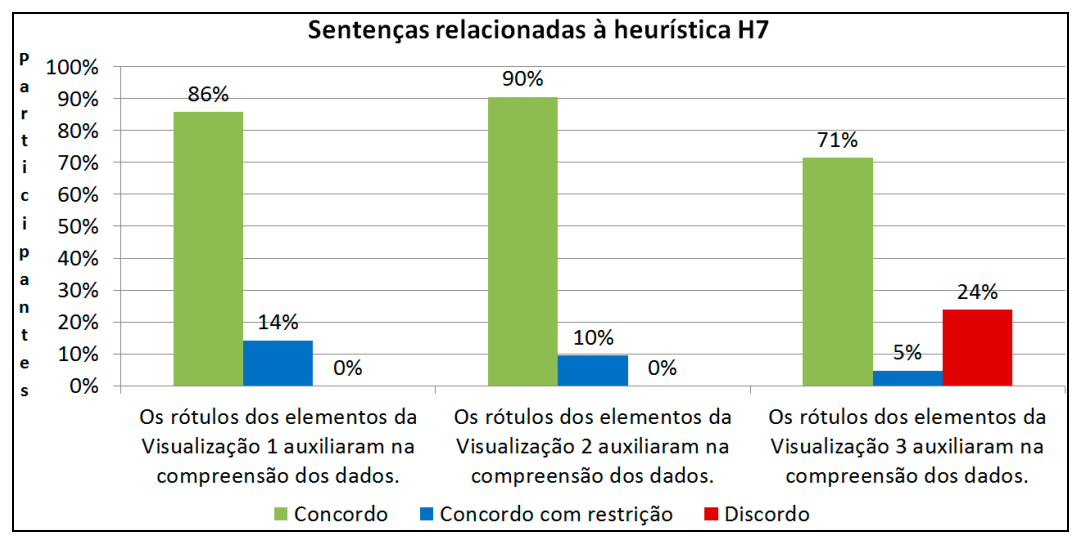

Figura 8. Sentenças relacionadas à heurística $\mathbf{H 7}$.

O tópico transparência dos dados também foi avaliado durante o experimento. A Visualização 1 não atendia às heurísticas: H2, H3, H5 e H6. Segundo 76\% dos participantes, as dificuldades encontradas nessa visualização prejudicaram a transparência dos dados. Já a Visualização 2 atendia às heurísticas: H1, H2, H3, H6 e H7. Para esta visualização, somente $33 \%$ das pessoas ficaram com a impressão de que alguma informação não foi revelada na visualização 2 . Além disso, $71 \%$ concordaram que a transparência dos dados é influenciada pela impressão que se tem das informações não reveladas, conforme mostra a Figura 9.

Em torno de $77 \%$ dos participantes concordaram que os dados ficaram mais transparentes na Visualização 3 do que na Visualização 4. A diferença entre estas visualizações é que a Visualização 3 atendia a todas as heurísticas e a Visualização 4 atendia apenas a heurística H7. Os resultados apresentados nas quatro visualizações sugerem que a percepção dos usuários sobre transparência dos dados é influenciada pelas características da técnica. 


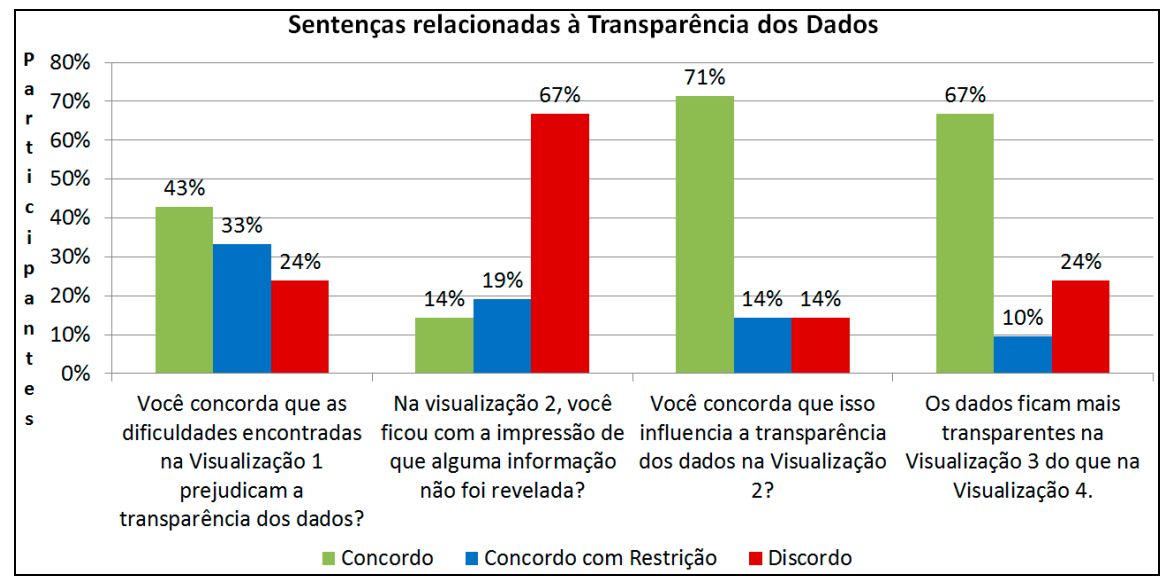

Figura 9. Sentenças relacionadas à transparência.

\section{Considerações Finais}

O objetivo deste trabalho foi analisar a literatura sobre visualização de dados para definir um conjunto de heurísticas de forma a facilitar a análise da aplicabilidade de técnicas de visualização no contexto governamental. As heurísticas não foram definidas restritamente para dados governamentais abertos. O conjunto de heurísticas também pode ser utilizado para avaliar as técnicas de visualização que representam outros tipos de dados. Os dados governamentais foram utilizados para mostrar que as características da visualização influencia a percepção que os usuários têm em relação à transparência dos dados.

Os resultados do experimento deste trabalho mostram que as heurísticas são relevantes e que podem auxiliar na aplicabilidade das técnicas de visualização. Os resultados também sugerem que as características da visualização influenciam a percepção dos usuários sobre transparência dos dados.

Como trabalho futuro sugere-se a extensão do conjunto de heurísticas considerando aspectos que não foram tratados neste trabalho. Outra meta futura é a realização de experimentos com um número maior de técnicas e participantes.

\section{Referência Bibliográfica}

Aló, C. C. (2009) "Uma abordagem para transparência em processos organizacionais utilizando aspectos". Dissertação de Doutorado em Informática. Pontifícia Universidade Católica do Rio de Janeiro, Rio de Janeiro.

Few, S. (2008) "Practical Rules for Using Color in Charts". Disponível em: $<$ http://www.perceptualedge.com/library.php\#Articles>. Acesso em 05 de out. 2012.

Forsell, C. (2012) "Evaluation in Information Visualization: Heuristic Evaluation". In: Information Visualisation (IV), 2012 16th International Conference on, vol., no., pp.136-142, 11-13 July 2012.

Forsell, C., e Johansson, J. (2006) "An heuristic set for evaluation in information visualization". In: Proceeding AVI'10 Proceedings of the International Conference on Advanced Visual Interfaces. Pages 199-206. ACM, New York, USA. 
Luzzardi, P. R. G. (2003) "Critério de Avaliação de Técnicas de Visualização de Informações Hierárquicas”. Dissertação de Doutorado. Programa de Pós-Graduação em Computação. Universidade Federal do Rio Grande do Sul, Porto Alegre, Brasil.

Nielsen, J., e Mack, R.L. (1994) "Usability Inspection Methods". Disponível em: $<$ http://www.behavioristics.com/downloads/usabilityinspectionmethods.pdf $>$. Acesso em 11 de dez. 2012.

Paula, M. M. V. de, Ribeiro, F. C., Chaves, M., Rodrigues, S. A. e Souza, J. M. de. (2011) “A Visualização de Informação e a Transparência de Dados Públicos", In: Simpósio Brasileiro de Sistemas de Informação. Salvador, Bahia.

Serie 1. (2012) "Recursos do FGTS aplicados em Habitação". Disponível em: $<$ https://i3gov.planejamento.gov.br/dadosgov/xml/serie/228.xml $>$. Acesso em $25 \mathrm{de}$ nov. 2012.

Serie 2. (2012) "Quantidade de escolas beneficiadas com o programa Escola Aberta". Disponível em: <https://3gov.planejamento.gov.br/dadosgov/xml/serie/1636.xml>. Acesso em 25 de nov. 2012.

Serie 3. (2012) "Pessoas atendidas pelo programa Luz Para Todos". Disponível em: $<$ https://i3gov.planejamento.gov.br/dadosgov/xml/serie/60.xml $>$. Acesso em $25 \mathrm{de}$ nov. 2012.

Silva, D. B. (2010) "Transparência na esfera pública interconectada". Dissertação de Mestrado em Comunicação - Faculdade Cásper Líbero, São Paulo.

Sorj, B. (2003) "Brasil@povo.com: a luta contra a desigualdade na sociedade da informação". Jorge Zahar Editor Ltda. Ed. Rio de Janeiro, RJ.

Souza, C. L. de, Guimarães, C. (2009) "Sistemas de Informação versus usuários". eXacta, Belo Horizonte, v.2, n.2, 2009. ISBN: 1984-3151.

Tory, M. e Moller, T. (2005) "Evaluating Visualizations: Do Expert Reviews Work". IEEE Computer Graphics and Applications, 25(5):8-11, September/October.

Tufte, E. R. (2001) "The Visual Display of Quantitative Information and Envisioning Information”, Graphics Press, PO Box 430, Cheshire, CT 06410.

Valiati, E. R. de A. (2008) "Avaliação de Usabilidade de Técnicas de Visualização de Informações Multidimensionai"s. Dissertação de Doutorado. Programa de PósGraduação em Computação. Universidade Federal do Rio Grande do Sul, Porto Alegre, Brasil.

Yamaguchi, J. K. (2010) "Diretrizes para a escolha de técnicas de visualização aplicadas ao processo de extração de conhecimento". Dissertação de Mestrado em Ciência da Computação - Universidade Estadual de Maringá.

Yau, N. (2010) "7 Basic Rules for Making Charts and Graphs". Disponível em: $<$ http://flowingdata.com/2010/07/22/7-basic-rules-for-making-charts-and-graphs/>. Acesso em 06 de out. 2012.

Zuk, T., Schleiser, L., Neuman, P., Hancook, M.S. e Carpendale, S. (2006) "Heuristics for Information Visualization Evaluation”. In Proc BELIV'06 ACM Press1-6. 